\title{
The Formation of Dental Microbial Biofilm and Plaque Associated with the Presence of Orthodontic, Taif, and KSA
}

\author{
* Sherifa Mostafa M. Sabra \\ *Microbiology Br., Biology Dept., Science Coll., Taif Uni., KSA
}

\begin{abstract}
Study was carried from Taif private dental center, start after got an agreement from center owner and the dentist. Understudy pts. were (No.=55), aged (15-30yrs.). All wearing a fixed orthodontic were divided into control group 15pts., with received oral and diet hygiene at dental center and understudy group 40pts., with received orders for oral and diet hygiene to make it at home. Fixed orthodontic stages investigation at a period of baseline and stages at $\left(1^{\text {st }}, 2^{\text {nd }}, 3^{\text {rd }}, 4^{\text {th }}, 5^{\text {th }}\right.$, and $6^{\text {th }}$ month). Getting consent forms agreement, arranged dental clinic regular visits for all understudy pts. Baseline were at the day of orthodontic fixation for both groups, followed up by period of 6months. Microbial examination of Dental Microbial Biofilm had done as well as Plaque Index regularly every month.Incidence of CFUs/ml saliva for control and understudy group during observation period 6months, there were in control group as 6.7, 6.7, 6.7, 13.3, 13.3, and 20\%, but in understudy group were $37.5,40,45,50,55$, and $57.5 \%$ respectively, there were appeared with CFUs/mlas $\left(10^{5}-10^{6}\right),\left(10^{6}\right.$ $\left.-10^{7}\right),\left(10^{7}-10^{8}\right),\left(10^{8}-10^{9}\right),\left(10^{9}-10^{10}\right)$, and $\left(10^{10}-10^{11}\right)$, respectively. Incidence of Plaque Index (PI) for control and understudy group during observation period 6months, there were in control group as 6.7, 13.3, 20, 20, 26.7 and 26.7\%, but in understudy group 37.5, 40, 50, 52.5, 57.5 and 60\%, that were appear with Plaque Index as (0-1), (0-1), (0-1), (1-2), (1-2) and (2-3) respectively.
\end{abstract}

Key words :Dental, Microbial Biofilm, Pts., Orthodontic, CFUs/ml, Plaques Index (PI).

\section{Introduction:}

Orthodontic treatment is becoming increasingly popular. The region of the tooth surface around the brackets is prone to adhesion of oral bacteria and subsequent biofilm formation. Oral biofilm, or "dental plaque", is difficult to remove and regular brushing is often insufficient to remove plaque from retention sites, such as the vulnerable bracket-adhesive-enamel junction, the sensitive region between brackets and the gingival. Biofilms on dental hard and soft tissues, as well as on different biomaterials employed for restoration of function in the oral cavity, are the main cause of dental disease ${ }^{[1]}$. Orthodontic biofilms are diverse communities of micro-organisms on dental hard and soft tissues and dental biomaterials, embedded in an extracellular matrix of polymers of host and microbial origin.Conditioning film formation: a salivary conditioning film, known in dentistry as the "acquired pellicle", forms immediately after cleaning or introducing new surfaces into the oral cavity. Reversible adhesion: an interplay of attractive Lifshitz-Van der Waals forces and electrostatic repulsion between bacteria and substratum surfaces yields an initially reversible adhesion. Since conditioning films form more rapidly than bacteria can be transported to the surface, bacteria mostly adhere to a salivary conditioning film and seldom to a bare substratum surface ${ }^{[2]}$. Factors influencing orthodontic biofilm formation, orthodontics adhesives, fixed appliances including brackets, ligating devices and arch wires, or removable acrylic plates are important factors influencing orthodontic biofilm formation. Adhesives. Composite resins for orthodontic bonding are in direct contact with the vulnerable enamel surface and their properties with respect to bacterial adhesion may be more important than of other orthodontic materials. In general, excessive composite resin at the bracket-enamel-adhesive junction is prone to bacterial adhesion, especially since polymerization shrinkage may yield a gap with a width of up to $10 \mathrm{~m}$ at the adhesive-enamel interface where bacteria find themselves protected against oral cleansing forces and antibacterial components of toothpastes and mouth rinses. Its harvesting different strains of Strept. spp., and Candida ${ }^{[3]}$. Healthy tooth surfaces and gingivae tend to only be associated with this first phase of biofilm development. It consists of an initial few layers (1-20) of mostly Gram positive Cocci bacteria, followed by some Gram positive rods and filaments and a very small amount of Gram negative Cocci. The Gram positive Cocci species involved in this conditioning layer include, but are not limited to, Strept. mutans, Strept. mites, Strept.sanguis, Strept. oralis and Staph. epidermidis. Gram negative rod and filament species include Actinomyces spp., and Corynebacterium spp., Veillonella spp. and Neisseria spp. are able to adhere to the non-exfoliating hard tooth surfaces. The early colonizers are also able to survive in the high oxygen concentrations present in the oral cavity, without having much protection from other bacteria. Thus, this thin, initial biofilm is almost always present on the tooth surface as it forms immediately after cleaning ${ }^{[4]}$. In the very early stages, the predominant tooth colonizers were found to be Actinomyces spp. The relative proportion of Strept., in particular Strept. mites and Strept. oralis, increased at the expense of Actinomyces spp. between (26 hrs.), while the absolute level of Actinomyces remained unaltered. Periodontal pathogens such as Bacteriodes 
spp., Porphyromonas gingivalis and Treponemadenticola, Actinobacillus Actinomycete mcomitans were present in extremely low levels at all the examined time intervals in this healthy group of subjects. It detailed insight into the bacterial population shifts occurring within the first few hours of biofilm formation and show that the early colonizers of the tooth surface predominantly consist of beneficial micro-organisms. The early colonizers of dental plaque are of great importance in the succession stages of biofilm formation and its overall effect on the oral health of the host ${ }^{[5]}$. Complicated appliance designs with loops and auxiliary arch wires create areas that are difficult to clean and may therefore enhance biofilm formation. The inter-bracket part of arch wires is relatively distant from the enamel surface and gingival tissues, and biofilms formed here may also be considered relatively harmless to the enamel and gingival tissues. Moreover, biofilms on these parts are easier to remove by brushing, compared with those formed on brackets, adhesives, and ligating devices. Biofilms on the arch wires ligated in the bracket slot may however, compromise the efficiency of the sliding mechanics ${ }^{[6]}$. Biofilms have been observed on acrylic base-plates after one week of wear ${ }^{[7]}$. Reduce the self-clearance by saliva, change the composition of the oral flora, increase the amount of oral biofilm formed, and the colonization of oral surfaces by cariogenic, and periodontopathogenic bacteria. These factors strongly complicate orthodontic treatment, and illustrate that the need for oral biofilm control is even greater during orthodontic treatment than usual. Despite current preventive measures to control biofilm formation during orthodontic treatment, the prevalence of biofilm-related problems has remained high. Consequences of orthodontic biofilms Enamel demineralization, enamel demineralization surrounding brackets is the most common side-effect of orthodontic treatment, affecting around $50 \%$ of all patients. White spot lesions can develop rapidly in susceptible individuals within the first month of treatment, and can remain visible many years after deboning, or in severe cases appear as a permanent enamel $\mathrm{scar}^{[8]}$. Fixed retainers are in direct contact with the enamel surface and cannot be removed for extensive cleaning like removable ones. Therefore they are generally considered to yield increased biofilm formation with negative consequences with respect to gingival inflammation ${ }^{[9]}$. The composition of orthodontic biofilms varies during the course of treatment. Placement of an orthodontic appliance increases not only the amount of biofilm, but also the prevalence of cariogenic bacteria ${ }^{[10]}$. Once biofilm formation has passed the stage of initial adhesion, environmental factors like $\mathrm{pH}$ and nutrient availability often play a more decisive role in the final amount of biofilm formed than differences in initial adhesion that can be achieved by altering the biomaterials surface properties, which yields limited reductions in microbial adhesion numbers of utmost a factor of ten. Under clinical conditions, roughness overrides all beneficial effects of biomaterials coatings, especially in supra-gingival regions ${ }^{[11]}$. Co-adhesion of micro-organisms to already adhering, early colonizers, which is also mediated by long-range, attractive Lifshitz-Van der Waals forces and highly specific stereochemical interactions at close approach ${ }^{[12]}$. Moreover, bacterial adhesion forces to composite resin, which often have a rougher surface than enamel or brackets, were stronger than to brackets or saliva-coated enamel, and depended on the bacterial strain involved. The severity of demineralization can range from white spot lesions to cavitation upon bracket removal, which can occur on both vestibular and lingual surfaces, with the most affected sites being the bracket-adhesive-enamel junction and the most affected teeth being the first molars, upper lateral incisors, and lower canines ${ }^{[13]}$. Biofilm formation on the brackets themselves is less harmful than when formed at the bracket-enamel-adhesive junction. Ligating Devices. Steel ligature wires and elastomeric rings are the most frequently used devices for ligating orthodontic wires into brackets before the introduction of self-ligating brackets. Between elastomeric rings and steel ligature wires, no difference was found regarding biofilm weight or biofilm-related clinical indices. However, elastomeric rings are related to a higher possibility of enamel demineralization, and are not recommended in patients with poor oral hygiene ${ }^{[14]}$. Oral biofilms in general comprise about $80 \%$ water and $20 \%$ of solid phase components including proteins, carbohydrates, fat, and inorganic components. The bacterial diversity in the oral cavity is estimated to include at least 800 different species, consisting of a wide variety of Gram positive and Gram negative bacteria, such as facultative anaerobes and obligatory anaerobes species. Mechanism of biofilm formation oral biofilms accumulate through sequential and ordered colonization of oral surfaces by the different strains and species present in the oral cavity ${ }^{[15]}$.Physico-chemical properties, such as hydrophobicity, surface charge and surface free energy are generally believed to play an important role, particularly in the initial stages of bacterial adhesion to surfaces, although no physico-chemical explanation of microbial adhesion to surfaces has been forwarded that goes beyond validity for selected collections of strains and surfaces. Also the removal of biofilm from rougher surfaces is more difficult than from smooth surfaces. Consequently, in vivothe natural oral cleansing forces are less effective in removing oral biofilm. Moreover, rough surfaces offer protection against oral shear forces, but also provide a protective shelter against environmental attacks such as by anti-bacterial oral health care components. The increase in roughness of the appliance materials due to biofilm is especially troublesome, since a rougher surfaces promote biofilm formation ${ }^{[16]}$. For the microbial count, it is note that caries-free subjects usually present with $<10^{5} \mathrm{CFUs} / \mathrm{ml}$ saliva. This level was considered the cut-off value to record the data. Different trend in the microbial colonization for the two treated groups was observed, as the group treated with conventional appliances showed a drastic increase of subjects CFUs $>10^{5}$ after 3 months from the beginning of 
treatment, $60 \%$ with a significant reduction after other 6 months, when they showed lower values respect to the other two groups $20 \%$. The assortment of the various species of bacteria in the oral microbial population observed during the early stages of the orthodontic treatment is not necessarily the same as that observed after a few months of orthodontic treatment ${ }^{[17]}$.

Aim of the work: Investigation the adhesion forces of different oral bacterialstrains to orthodontic materials with a biofilm formation and Plaque Index level consisting for long run. That, having identified the adhesive as the site of strongest adhesion forces, adjacent inaddition to the vulnerable enamel surface.

\section{Materials and Methods:}

Materials:Study Field were from Taif private dental center, start after got an agreement from center owner and dentists.Understudy pts. were (No.=55), aged (15-30yrs.).All wearing a fixed orthodontic were divided into control group 15pts., with received oral hygiene at dental center. understudy group 40pts., with received orders for oral hygiene to make it at home.Stages was as:fixed orthodontic stages investigation ata period of baseline and stages at $\left(1^{\text {st }}, 2^{\text {nd }}, 3^{\text {rd }}, 4^{\text {th }}, 5^{\text {th }}, 6^{\text {th }}\right.$ month $)$. Group preparation was as, got consent agreement, arrange dental clinic regular visit for control and understudy pts.

Methods:Data collection were for studypatients, must be healthy, free of diseases, don't taking any medication. Baselinewere at the day of orthodontic fixation for both group, followed up by period of 6 months. The determination of dental biofilm regularly every month and bacterial examination will be done regularly as well.Physical characters of teeth done on all patients every month. Collection of samples, biological sample collection and processing. All biofilm samples were collected by one investigator, after pumicing tooth surfaces, the teeth were isolated from buccal/labial mucosa with cotton rolls during the entire sampling procedure to avoid contact between tooth surfaces and oral mucosa.Microbial pattern for (isolation, identification, CFUs/ml), the saliva samples were inoculated on two different selective agar plates, to record coloniescounts in saliva by means of selective media. The agar plates were incubated at $37^{\circ} \mathrm{C}$ for 2 days in a $\mathrm{CO}_{2}$ atmosphere, following which the totalcount was performed. counts CFUs/mlsaliva ${ }^{[18]}$. Plaque index (PI) assessment, it's as developed assesses the thickness of plaque at the cervical margin of the tooth (closest to the gum). Four areas, distal, labial or buccal, mesial, and lingual or palatal, were examined. Each tooth was dried and examined visually using a mirror, and an explorer, and adequate light. The explorer was passed over the cervical third to test for the presence of plaque. Four different scores are possible. Zero indicates no plaque present, lindicates a film of plaque present on the tooth, 2represents moderate accumulation of soft deposits in the gingival pocket or on the tooth that can be seen by the naked eye, 3represents an abundance of soft matter within the pocket or on the tooth. Each area of each tooth is assigned a score from (0-3). Scores for each tooth were totaled and divided by the four surfaces scored. To determine a total PI for an individual, the scores for each tooth are totaled and divided by the number of teeth examined. Four ratings may then be assigned: $(0)=$ excellent, $(0-1)=$ good, $(1-$ 2)=fair, $(2-3)=$ poor $^{[19]}$.

Data Analysis:The data were recorded and entered into Microsoft excel sheet and analyzed ${ }^{[20]}$.

III. Results and discussion:

Table and figure1: Incidence of CFUs/ml saliva for control and understudy group during observation period 6months

\begin{tabular}{|c|c|c|c|c|c|c|c|}
\hline Month & Baseline & 1st & 2nd & 3rd & 4th & 5th & 6th \\
\hline $\begin{array}{l}* \text { CFUs/ml } \\
\text { saliva }\end{array}$ & $\left(<10^{5}\right)$ & $\begin{array}{c}\left(10^{5}-10^{6}\right) \\
+\end{array}$ & $\begin{array}{c}\left(10^{6}-\right. \\
\left.10^{7}\right) \\
++\end{array}$ & $\begin{array}{l}\left(10^{7}-\right. \\
\left.10^{8}\right) \\
+++\end{array}$ & $\begin{array}{c}\left(10^{8}-10^{9}\right) \\
++++\end{array}$ & $\begin{array}{c}\left(10^{9}-\right. \\
\left.10^{10}\right) \\
++++ \\
+\end{array}$ & $\begin{array}{c}\left(10^{10}-\right. \\
\left.10^{11}\right) \\
+++++ \\
+\end{array}$ \\
\hline $\begin{array}{l}\text { Control } \\
\text { Group } \\
* \text { No.=15 }\end{array}$ & $\begin{array}{l}15 / 15 \\
100 \%\end{array}$ & $\begin{array}{l}1 / 15 \\
6.7 \%\end{array}$ & $\begin{array}{l}1 / 15 \\
6.7 \%\end{array}$ & $\begin{array}{l}1 / 15 \\
6.7 \%\end{array}$ & $\begin{array}{c}2 / 15 \\
13.3 \%\end{array}$ & $\begin{array}{c}2 / 15 \\
13.3 \%\end{array}$ & $\begin{array}{l}3 / 15 \\
20 \%\end{array}$ \\
\hline $\begin{array}{l}\text { Understudy } \\
\text { Group } \\
* \text { No.=40 }\end{array}$ & $\begin{array}{l}40 / 40 \\
100 \%\end{array}$ & $\begin{array}{c}15 / 40 \\
37.5 \%\end{array}$ & $\begin{array}{l}16 / 40 \\
40 \%\end{array}$ & $\begin{array}{l}18 / 40 \\
45 \%\end{array}$ & $\begin{array}{l}20 / 40 \\
50 \%\end{array}$ & $\begin{array}{l}22 / 40 \\
55 \%\end{array}$ & $\begin{array}{c}\mathbf{2 3 / 4 0} \\
57.5 \%\end{array}$ \\
\hline
\end{tabular}




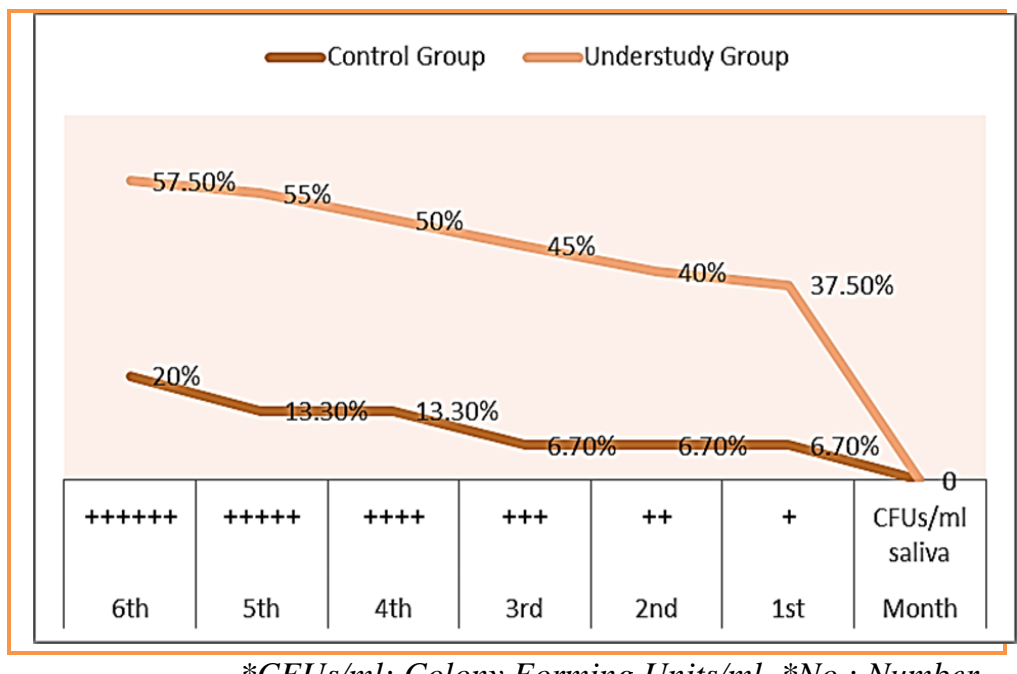

*CFUs/ml: Colony Forming Units/ml, *No.: Number

Table and figure 1 show incidence of CFUs/ml saliva for control and understudy group during observation period 6months, there were in control group as $6.7,6.7,6.7,13.3,13.3$, and $20 \%$, but in understudy group were as $37.5,40,45,50,55$, and $57.5 \%$ respectively, there were appeared with CFUs/ml were as $\left(10^{5}-\right.$ $\left.10^{6}\right),\left(10^{6}-10^{7}\right),\left(10^{7}-10^{8}\right),\left(10^{8}-10^{9}\right),\left(10^{9}-10^{10}\right)$, and $\left(10^{10}-10^{11}\right)$, respectively. Biofilms harvesting different strains of Strept. spp., and Candida ${ }^{[3]}$.It consists of an initial few layers (1-20) of, Gram positive Cocci species involved in this conditioning layer include, but are not limited to, Strept. mutans, Strept. mites, Strept.sanguis, Strept. oralis and Staph. epidermidis. Gram negative rod and filament species include Actinomyces spp., and Corynebacterium spp., Veillonella spp. and Neisseria spp. are able to adhere to the nonexfoliating hard tooth surfaces ${ }^{[4]}$.In the very early stages, the predominant tooth colonizers were found to be Actinomyces spp. The relative proportion of Strept., in particular Strept. mites and Strept. oralis, increased at the expense of Actinomyces spp. between (2-6hrs.), while the absolute level of Actinomyces remained unaltered. Periodontal pathogens such as Bacteriodes spp., Porphyromonas gingivalis and Treponemadenticola, Actinobacillus Actinomycete mcomitans were present in extremely low levels at all the examined time intervals in this healthy group of subjects. It detailed insight into the bacterial population shifts occurring within the first few hours of biofilm formation and show that the early colonizers of the tooth surface predominantly consist of beneficial micro-organisms ${ }^{[5]}$.The composition of orthodontic biofilms varies during the course of treatment. Placement of an orthodontic appliance increases not only the amount of biofilm, but also the prevalence of cariogenic bacteria ${ }^{[10]}$. For the microbial count, it is note that caries-free subjects usually present with $<10^{5}$ CFUs /ml saliva. This level was considered the cut-off value to record the data. Different trend in the microbial colonization for the two treated groups was observed, as the group treated with conventional appliances showed a drastic increase of subjects CFUs $>10^{5}$ after 3 months from the beginning of treatment, $60 \%$ with a significant reduction after other 6months, when they showed lower values respect to the other two groups $20 \%$. The assortment of the various species of bacteria in the oral microbial population observed during the early stages of the orthodontic treatment is not necessarily the same as that observed after a few months of orthodontic treatment ${ }^{[17]}$.

Table and figure2: Incidence of Plaque Index for control and understudy group during observation period 6months

\begin{tabular}{|c|c|c|c|c|c|c|c|}
\hline Month & Baseline & 1st & 2nd & 3rd & 4th & 5th & 6th \\
\hline $\begin{array}{l}\text { Plaque } \\
\text { Index }\end{array}$ & (0) & $\begin{array}{c}(0-1) \\
+\end{array}$ & $\begin{array}{c}(0-1) \\
+\end{array}$ & $\begin{array}{c}(0-1) \\
+\end{array}$ & $\begin{array}{c}(1-2) \\
++\end{array}$ & $\begin{array}{c}(1-2) \\
++\end{array}$ & $\begin{array}{c}(2-3) \\
+++\end{array}$ \\
\hline $\begin{array}{l}\text { Control } \\
\text { Group } \\
* \text { No.=15 }\end{array}$ & $\begin{array}{l}15 / 15 \\
100 \%\end{array}$ & $\begin{array}{c}1 / 15 \\
6.7 \%\end{array}$ & $\begin{array}{c}2 / 15 \\
13.3 \%\end{array}$ & $\begin{array}{l}3 / 15 \\
20 \%\end{array}$ & $\begin{array}{l}3 / 15 \\
20 \%\end{array}$ & $\begin{array}{c}4 / 15 \\
26.7 \%\end{array}$ & $\begin{array}{c}4 / 15 \\
26.7 \%\end{array}$ \\
\hline $\begin{array}{l}\text { Understudy } \\
\text { Group } \\
\text { *No.=40 }\end{array}$ & $\begin{array}{l}40 / 40 \\
100 \%\end{array}$ & $\begin{array}{c}15 / 40 \\
37.5 \%\end{array}$ & $\begin{array}{l}16 / 40 \\
40 \%\end{array}$ & $\begin{array}{c}\mathbf{2 0 / 4 0} \\
\mathbf{5 0 \%}\end{array}$ & $\begin{array}{r}21 / 40 \\
52.5 \%\end{array}$ & $\begin{array}{r}23 / 40 \\
57.5 \%\end{array}$ & $\begin{array}{c}24 / 40 \\
60 \%\end{array}$ \\
\hline
\end{tabular}




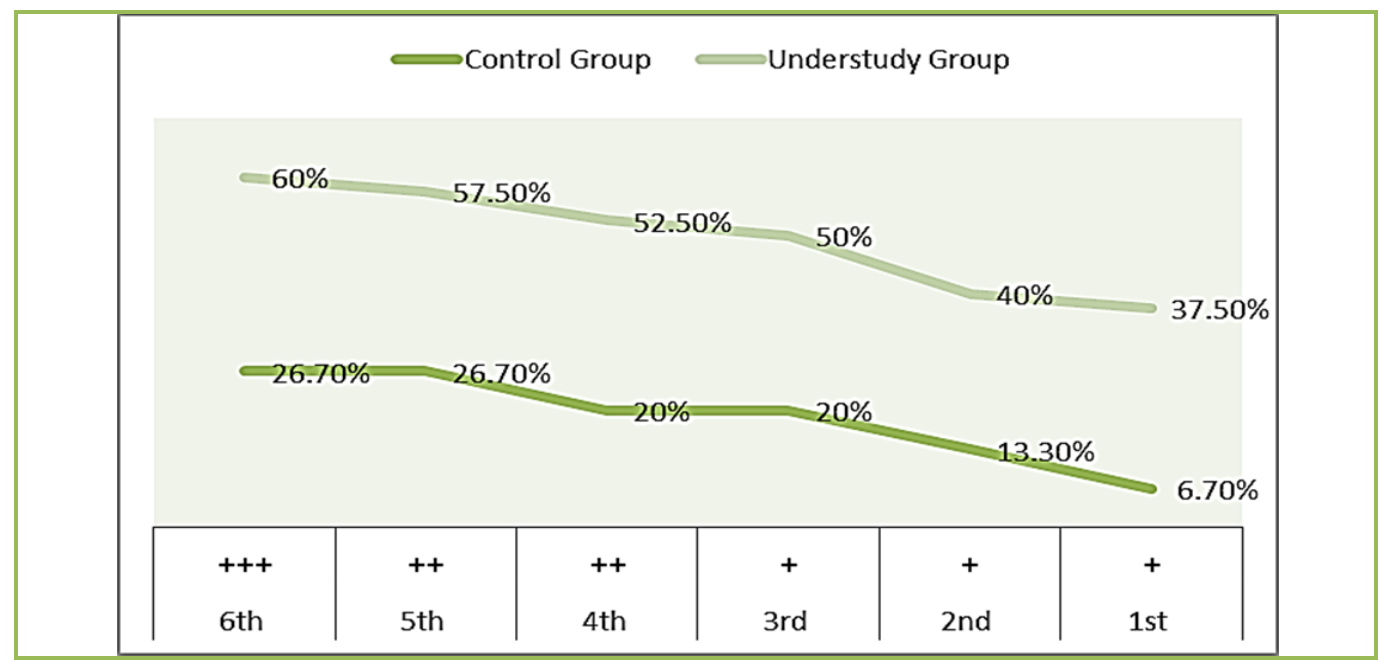

*No.: Number

Table and figure 2 showIncidence of Plaque Index for control and understudy group during observation period 6months, there were in control group $6.7,13.3,20,20,26.7$ and $26.7 \%$, but in understudy group were $37.5,40,50,52.5,57.5$ and 60\%, that appear with Plaque Index $(0-1),(0-1),(0-1),(1-2),(1-2)$ and (2-3) respectively.Oral biofilm, or "dental plaque", is difficult to remove and regular brushing is often insufficient to remove plaque from retention sites, such as the vulnerable bracket-adhesive-enamel junction, the sensitive region between brackets and the gingival ${ }^{[1]}$. Conditioning film formation: a salivary conditioning film, known in dentistry as the "acquired pellicle", forms immediately after cleaning or introducing new surfaces into the oral cavity $^{[2]}$. Biofilms have been observed on acrylic base-plates after one week of wear ${ }^{[7]}$. Consequences of orthodontic biofilms Enamel demineralization, enamel demineralization surrounding brackets is the most common side-effect of orthodontic treatment, affecting around $50 \%$ of all patients. White spot lesions can develop rapidly in susceptible individuals within the first month of treatment, and can remain visible many years after deboning, or in severe cases appear as a permanent enamel scar ${ }^{[8]}$. The severity of demineralization can range from white spot lesions to cavitation upon bracket removal, which can occur on both vestibular and lingual surfaces, with the most affected sites being the bracket-adhesive-enamel junction and the most affected teeth being the first molars, upper lateral incisors, and lower canines ${ }^{[13]}$. To determine a total PI for an individual, the scores for each tooth are totaled and divided by the number of teeth examined. Four ratings may then be assigned: $(0)=$ excellent, $(0-1)=$ good, $(1-2)=$ fair, $(2-3)=$ poor $^{[19]}$.

\section{Conclusions:}

Orthodontic appliances severely hamper biofilm control in the oral cavity, but once treatment has successfully ended, it creates a better condition for oral cleaning and strongly aids oral health by a properly aligned dentition. The negative side-effects of orthodontic treatment as related to biofilms, such as white spot lesions and gingivitis, compromise the facial esthetics aimed for by the treatment after an often lengthy and costly course of orthodontic treatment. In severe cases, orthodontic appliances have to be removed before the treatment goal has been reached. Most orthodontists are aware of these problems, but yet effective preventive programs are lacking. To date, study biofilm, plaque, decalcification, demineralization, white spot lesion, gingivitis, or gingival inflammation. Considering the prevalence and seriousness of the consequences of orthodontic biofilms, more attention from orthodontic societies is needed, both in terms of research as well as patient education about the potential side-effects of orthodontic treatment. A combination of proper oral hygiene instruction, preventive measures, early diagnosis, and timely treatment would contribute greatly to the management of orthodontic biofilms. Moreover, new orthodontic materials should be developed attracting less biofilm and with appropriate anti-bacterial properties.

\section{Acknowledgement:}

Best regards and apparitions sent to dental center owner and dentists, as well more grateful to patients agreed and shared in this work. 


\section{References:}

[1] Filoche, S., Wong, L. andSissons, C., 2010. Oral biofilms: emerging concepts in microbial ecology. J. Dent. Res., 89:8-18.

[2] Marsh, P., Moter A. and Devine, D., 2010. Dental plaque biofilms: communities, conflict and control Periodontal., 55:16-35.

[3] Sukontapatipark, W., El-Agroudi, M., Selliseth, N., Thunold, K. and Selvig, K., 2001. Bacterial colonization associated with fixed orthodontic appliances. A scanning electron microscopy study. Eur. J. Orthod., 23:475-484.

[4] Sbordone, L. and Bortolaia, C., 2003. Oral microbial biofilms and plaque-related diseases: microbial communities and their role in the shift from oral health to disease, Cline. Oral Invest., 7:181-188.

[5] Li, E., Helmerhorst, C., Leon,1 R., Troxler, T., Yaskell, A., Haffajee, S., Socransky, S. and Oppenheim, F., 2004. Identification of early microbial colonizers in human dental biofilm. J. Applied Micro., 97:1311-1318.

[6] Eliades, T. and Bourauel, C., 2005. Intraoral aging of orthodontic materials: the picture we miss and its clinical relevance. Am. J. Orthod. Dentofacial. Orthop., 127:403-412.

[7] Lessa, F., Enoki, C. Ito I., Faria, G., Matsumoto, M. and Nelson-Filho, P., 2007. In-vivo evaluation of the bacterial contamination and disinfection of acrylic baseplates of removable orthodontic appliances. Am. J. Orthod.Dentofacial. Orthop., 131:711-707.

[8] Ogaard, B., 2008. White spot lesions during orthodontic treatment: mechanisms and fluoride preventive aspects Seminars in Orthodontics, 14:183-193.

[9] Levin, L., Samorodnitzky-Naveh, G. and Machtei, E., 2008. The association of orthodontic treatment and fixed retainers with gingival health. J. Periodontal., 79:2087-2092.

[10] Al-Mulla, A., Kharsa, S., Kjellberg, H. and Birkhed, D., 2009. Caries risk profiles in orthodontic patients at follow-up using Cariogram. Angle. Orthod., 79:323-330.

[11] Subramani, K., Jung, R., Molenberg, A. and Hammerle, C., 2009. Biofilm on dental implants: a review of the literature. Int. J. Oral Maxillofac Implants., 24:616-626.

[12] Hojo, K., Nagaoka, S., Ohshima, T. and Maeda, N., 2009. Bacterial interactions in dental biofilm development. J. Dent. Res., 88:982-990.

[13] Mei, L., Busscher, H., Van der Mei, H., Chen, Y., De Vries, J. and Ren, Y., 2009. Oral bacterial adhesion forces to biomaterial surfaces constituting the bracket-adhesive-enamel junction in orthodontic treatment. Eur. J. Oral. Sci., 117:419-426.

[14] Gameiro, G., Nouer, D., Cenci, M. and Cury. J., 2009. Enamel demineralization with two forms of archwire ligation investigated using an in situ caries model--a pilot study. Eur. J. Orthod., 31:542-546.

[15] Filoche, S., Wong, L. and Sissons, C., 2010. Oral biofilms: emerging concepts in microbial ecology. J. Dent. Res., 89:8-18.

[16] Busscher, H., Rinastiti, M., Siswomihardjo, W. and Van der Mei, H., 2010. Biofilm formation on dental restorative and implant materials. J. Dent. Res., 89:657-665.

[17] Stefano, M., Enrico, M. and Maria, R., 2013. In-office bacteria test for a microbial monitoring during the conventional and self-ligating orthodontic treatment, Head \& Face Med., 3: 9-17.

[18] Aas J., Griffen A., Dardis S., Lee A., Olsen I., Dewhirst F., Leys E. and Paster B., 2008. Bacteria of dental caries in primary and permanent teeth in children and young adults. J. Cline. Micro., 46:1407-1417.

[19] Löe, H., 1967. The Gingival Index, the Plaque Index and the Retention Index Systems. J. Periodontology, 38:610-616.

[20] Coulombier, D., Fagan, R., Hathcock, L. and Smith, C., 2001. Epi Info 6 Version 6.04.A Word Processing, Database and Statistical Program for Public Health. Centers for Disease Control and Prevention, Atlanta, Delaware, USA. 